\title{
Les dimensions silencieuses de l'acceptabilité sociale au Nunavik
}

Le cas d'Aupaluk

\section{The Silent Dimensions of Social Acceptability in Nunavik}

The Case of Aupaluk

\section{Las dimensiones silenciosas de la aceptación social en Nunavik El caso de Aupaluk}

\section{Julie Fortin}

Volume 49, numéro 2, 2019

Le consentement préalable, libre et éclairé : du principe à la mise en oeuvre en contexte canadien

URI : https://id.erudit.org/iderudit/1070760ar

DOI : https://doi.org/10.7202/1070760ar

Aller au sommaire du numéro

Éditeur(s)

Recherches amérindiennes au Québec

ISSN

0318-4137 (imprimé)

1923-5151 (numérique)

Découvrir la revue

Citer cet article

Fortin, J. (2019). Les dimensions silencieuses de l'acceptabilité sociale au Nunavik : le cas d'Aupaluk. Recherches amérindiennes au Québec, 49(2), 73-84. https://doi.org/10.7202/1070760ar

\section{Résumé de l'article}

Aupaluk est le plus petit village du Nunavik, avec une population d'environ 200 personnes. Depuis très longtemps, ce territoire est convoité par les compagnies minières en raison de sa concentration élevée en minéraux, mais surtout en fer. Aupaluk est dans la mire de deux compagnies minières juniors canadiennes : Oceanic Iron Ore et Nickel North Exploration. Ces projets suscitent des attentes et des craintes, notamment en raison de leur localisation à proximité du village et du peu d'information disponible. À l'automne 2017, vingt-trois entretiens semi-dirigés ont été menés avec les membres de la communauté afin d'analyser leur position par rapport au développement minier. L'analyse de l'auteure fait ressortir de nombreux obstacles qui empêchent les membres de la communauté d'exprimer leurs préoccupations par rapport aux projets, soit des processus de consultation et de communication inadéquats, la présence de claims miniers historiques dans la région, l'insécurité alimentaire et une certaine pression sociale.
Tous droits réservés @ Recherches amérindiennes au Québec, 2020

Ce document est protégé par la loi sur le droit d'auteur. L'utilisation des services d'Érudit (y compris la reproduction) est assujettie à sa politique d'utilisation que vous pouvez consulter en ligne.

https://apropos.erudit.org/fr/usagers/politique-dutilisation/ 


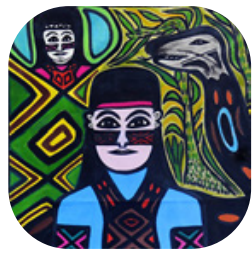

Les dimensions silencieuses

de l'acceptabilité sociale au Nunavik

Le cas d'Aupaluk

\author{
Julie Fortin \\ Candidate au \\ doctorat en \\ communication \\ publique, \\ Université Laval, \\ Québec
}

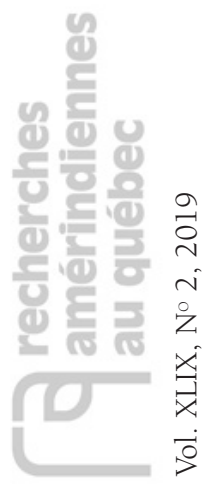

1

ICHÉ SUR LA CÔTE OUEST de la baie d'Ungava, Aupaluk est le plus petit village du Nunavik, avec une population d'environ 200 personnes. La riche concentration en fer du sol lui a valu son nom, qui signifie « là où la terre est rouge ». En effet, Aupaluk est situé à l'extrémité nord de la fosse du Labrador, une ceinture géologique de 1100 kilomètres de long qui traverse le Québec et le Labrador, et se déploie de Sept-îles à Aupaluk. Depuis très longtemps, ce territoire est convoité par les compagnies minières en raison de sa concentration élevée en minéraux, surtout en fer de grande qualité. Aupaluk est actuellement dans la mire de deux compagnies minières juniors (voir Deshaies 2011) canadiennes: Oceanic Iron Ore et Nickel North Exploration, le projet Hawk Ridge étant quant à lui en suspens depuis 2016. En dépit des bénéfices économiques que le développement minier peut engendrer, l'ampleur des projets, leur proximité avec le village et le manque d'information à leur sujet divisent la population et suscitent des inquiétudes chez les Aupalumiut. Cet article nous permettra de mieux comprendre les différents obstacles qui limitent la capacité de négociation et de délibération des Aupalumiut et qui sont notamment liés aux relations de pouvoir entre les acteurs dans le cadre des projets miniers au stade d'exploration.

\section{Problématique}

Avec le jugement Strateco (voir Guikawuihé 2018; Bourgeois 2017; Landry et Mateu 2017), l'acceptabilité sociale est un motif qu'on peut désormais invoquer pour ne pas autoriser un projet de développement des ressources dans un milieu donné. Qu'elle soit motivée par un souci de bonnes pratiques de gouvernance ou encore dans une optique de gestion du risque, l'acceptabilité sociale fait l'objet d'une attention grandissante de l'État et des promoteurs, comme en témoigne la publication de nombreux guides sur le sujet (MRN 2016; CPEQ 2012). Elle repose à la fois sur des facteurs propres au milieu d'accueil (valeurs, croyances et attentes) et sur les risques, les impacts et les bénéfices anticipés pour les populations concernées et leur milieu. Elle revêt également une forte dimension relationnelle, qui se traduit par le degré de confiance envers les institutions et les promoteurs (Gauthier 2015; Batellier 2015; Gendron 2014; Maillé 2012). Surtout, elle constitue une forme d'action publique, un « travail politique » que réalise une population donnée pour déterminer la pertinence et la légitimité d'un projet (Fortin et Fournis 2015). Cette action publique prend la forme d'un «processus de négociation sociale » et est fortement « liée à la capacité 
collective et communautaire de délibération » (CaronMalenfant et al. 2013). On ne peut toutefois conclure qu'un projet est accepté socialement sur la seule base d'une absence de conflits ou de protestations ouvertes à son sujet. En effet, « [l]'inaction et le silence sont une forme de réponse du public, parfois choisie et/ou stratégique, parfois contrainte » (Batellier 2015 : 101). La prise en compte des dimensions «silencieuses » de l'acceptabilité sociale est d'autant plus difficile, car « le cadre plus large de la culture de gouvernance dans lequel s'insèrent les discours et les actions autour de l'acceptabilité sociale est rarement précisé » (Batellier 2015: 7), un élément particulièrement vrai en contexte autochtone. En effet, pour Campbell et Prémont (2017), l'acceptabilité sociale et la légitimité des opérations minières au Canada sont avant tout liées aux caractéristiques des droits miniers.

En effet, l'héritage colonial du régime minier canadien implique que les activités minières ont préséance sur tout autre usage du territoire, à l'exception des zones urbanisées, des parcs et des aires protégées. En effet, « contrairement aux régimes encadrant l'accès à d'autres ressources naturelles, telles les forêts, l'État ne joue qu'un rôle passif dans le processus d'acquisition des droits miniers », une situation qui implique qu'il ne peut « préférer au premier acquéreur d'un claim un prospecteur qui ferait montre d'une volonté et d'une capacité accrues d'exercer des activités d'exploration minière dans le respect de l'environnement et des populations avoisinantes » (Thériault $2010: 225$ ).

Malgré la facilité avec laquelle les promoteurs peuvent acquérir des claims miniers, ces derniers cherchent néanmoins à obtenir une licence sociale pour opérer et ce, pour limiter les risques financiers et préserver leur réputation. Ils veulent ainsi rendre leurs projets crédibles, légitimes et dignes de confiance (Prno et Slocombe 2012; Thomson et Boutilier 2011). La crédibilité s'acquiert lorsqu'un promoteur tient ses promesses et qu'il interagit honnêtement avec les membres d'une communauté, alors que la confiance se rapporte à des notions telles que l'écoute, le dialogue et la réciprocité et s'applique également au niveau de confiance que les membres d'une communauté entretiennent à l'égard des organisations qui les représentent (Koivurova et al. 2015). La difficulté d'opérationnaliser la licence sociale constitue toutefois un avantage pour les compagnies, qui sont libres d'interpréter ce qui constitue une telle «licence sociale », de même que les préoccupations des communautés qui sont considérées comme légitimes (Zahara, Keeling et Bell 2016).

En exposant les dimensions « silencieuses » de l'acceptabilité sociale en contexte inuit, notre article vise, d'une part, à faire ressortir les facteurs structurels et culturels qui nuisent à la capacité collective de délibération des membres de la communauté d'Aupaluk et, d'autre part, il constitue une tentative d'opérationnalisation du concept de licence sociale pour opérer du point de vue des communautés.

Notre question de recherche est donc la suivante: dans le cadre des projets miniers au stade d'exploration, dans quelle mesure la capacité de négociation et de délibération des communautés autochtones est-elle influencée par les relations de pouvoir entre les différents acteurs, la perception du promoteur ou la cohésion sociale de la communauté.

\section{Cadre théorique}

\section{L'INDUSTRIE ET LES REGISTRES « SILENCIEUX 》 DU POUVOIR}

Pour mieux saisir les différentes formes de pouvoir qui teintent les relations entre l'État, l'industrie minière et les communautés, nous utiliserons la typologie développée par John Allen (2003), qui définit le pouvoir comme étant relationnel et résultant des interactions sociales. Il distingue les formes plus explicites de pouvoir (domination, coercition et autorité) des registres « silencieux » du pouvoir (persuasion, séduction et manipulation). Ces derniers ont été adaptés aux stratégies utilisées par les industries extractives par Frederiksen et Himley (2019). Ainsi, le pouvoir de l'État est associé à l'autorité; il implique une conformité volontaire et suscite de la légitimité. Dans le contexte des activités extractives, il s'agira par exemple du régime minier et du processus d'évaluation environnementale. Frederiksen et Himley (2019) distinguent ensuite les trois formes de pouvoir « silencieuses » utilisées par l'industrie. D'abord, la persuasion comprend les stratégies visant à faciliter les négociations avec les communautés locales qui sont souvent incluses dans les Ententes sur les répercussions et les avantages (ERA), telles que les compensations financières, les emplois et les programmes de responsabilité sociale. Ensuite, la séduction, qui consiste pour le promoteur à faire la promotion d'un futur florissant où les activités extractives sont centrales et sont fortement liées aux notions de progrès et de modernité. Enfin, l'industrie peut utiliser des stratégies s'apparentant à de la manipulation ou à de la malhonnêteté et qui englobent notamment les promesses non tenues, les ententes non respectées et les attentes non rencontrées.

\section{LA CAPACITÉ D'EXPRESSION ET DE DÉLIBÉRATION DES COMMUNAUTÉS}

La capacité à résister à un projet et à le contester requiert certaines compétences, à la fois techniques et communicationnelles, chez les citoyens (Sauvé et Batellier 2011). Wolsink (2000) a mis de l'avant la notion de capital institutionnel du milieu social, qui fait référence à la capacité des acteurs à se faire entendre dans le débat et qui repose notamment sur la connaissance, la capacité relationnelle et la capacité de mobilisation. À noter toutefois que les pratiques de communication d'un promoteur peuvent contribuer à la détérioration du tissu social d'une communauté 
et ce, avant même que le projet ne soit autorisé (Maillé 2012). Au Canada, les Autochtones ont démontré leur importante capacité de mobilisation lors d'évènements ayant eu un fort impact politique : pensons notamment à la bataille médiatique et juridique qui a mené à la signature de la Convention de la Baie-James et du Nord québécois et, plus tard, au mouvement Idle No More. Toutefois, la capacité des communautés autochtones à exprimer leurs préoccupations au sein des mécanismes de consultation est très variable en raison du long historique colonial, auquel s'ajoutent les dynamiques de pouvoir à l'interne, qui sont moins souvent reconnues (Le Blanc 2009). Chez les Inuits par exemple, l'âge est un facteur déterminant dans la hiérarchie sociale, les aînés occupent souvent une place prépondérante dans le processus décisionnel inuit : « Les aînés sont non seulement respectés, mais ils sont aussi consultés pour leur sagesse et leurs conseils, en particulier sur les questions relatives à la terre et aux valeurs traditionnelles » (Stevenson 1993: 36). Ainsi, même s'ils ne prennent pas toujours part au processus décisionnel de façon officielle, leurs conseils sont souvent considérés comme ayant le plus de poids.

\section{Méthodologie}

Cet article constitue une étude de cas en relations publiques. Il vise à apporter une explication holistique à la façon dont différents aspects, influences et processus de communication sont interreliés au sein d'un même cas, ce qui permet d'effectuer une analyse approfondie d'un phénomène particulier, du point de vue des gens concernés. Surtout, une telle étude permet d'éclairer les « forces sociales plus larges et les régimes de pouvoir dans lesquels les cas sont imbriqués » (Piekkari et al. 2009, cités dans Daymon et Holloway 2010 : 115). En février 2015, nous avons participé à la réalisation d'un sondage sur le développement minier réalisé à Aupaluk par la Société Makivik; il s'agissait alors de notre premier contact avec les membres de la communauté. Un aperçu des résultats de ce sondage sera présenté plus loin. Par ailleurs, les données ${ }^{1}$ présentées ici ont été obtenues au moyen d'une analyse documentaire, d'observation et de vingt-trois entretiens semi-dirigés réalisés avec des membres de la communauté d'Aupaluk en novembre 2017. Parmi les participants figuraient des femmes, des hommes, des jeunes, des aînés et des membres de la Corporation foncière. Certains avaient une expérience préalable dans des projets miniers, mais ils étaient relativement peu nombreux. Les entretiens étaient d'une durée variant de 20 minutes à deux heures, et les questions portaient notamment sur la vision de la communauté en ce qui concerne le développement, le degré d'influence perçu, leur perception du leadership, de la Société Makivik et du promoteur, mais aussi des impacts du développement minier, de même que les émotions suscitées par les projets. Afin de conserver l'anonymat des participants, nous aurons recours à des pseudonymes pour présenter les extraits d'entretiens.

Lanalyse des données sera divisée en trois sections. La première exposera les relations de pouvoir à l'œuvre entre les différentes parties, soit le régime minier et les processus d'évaluation environnementale au Nunavik, de même que les stratégies déployées par le promoteur, soit les activités de lobbying, les relations diplomatiques et les politiques de responsabilité sociale. La deuxième section traitera du niveau d'acceptabilité sociale par rapport au projet Hopes Advance. Il sera ainsi question des risques perçus quant à la proximité du projet avec le village, de même que des bénéfices anticipés. La troisième section portera quant à elle sur le degré de confiance envers les institutions et les promoteurs, de même que les impacts sur la cohésion sociale et la capacité de délibération de la communauté. Enfin, ces données seront synthétisées afin d'évaluer la légitimité, la crédibilité ainsi que la confiance inspirée par le promoteur, conditions essentielles à l'obtention d'une licence sociale pour opérer.

\section{RÉGIME Minier et PROCESSUS d'ÉVALUATION DES PROJETS AU NUNAVIK}

La Convention de la Baie-James et du Nord québécois (CBJNQ) a entraîné la création de trois catégories de terres au Nunavik pour lesquelles les Inuits disposent de droits variables. Par exemple, les terres de catégorie 1 sont situées autour des villages et représentent une superficie de $3250 \mathrm{~m}^{2}$, dont les droits de surface sont détenus par les corporations foncières des communautés. Ainsi, toute activité minière qui se déroule en terres de catégories 1 requiert l'approbation de la corporation foncière ainsi qu'un référendum local. Les activités d'exploration et d'exploitation situées en terres de catégorie 2 nécessitent quant à elles une consultation préalable avec la Société Makivik (qui représente tous les Inuits du Nunavik pour les questions portant sur leurs droits collectifs et qui est l'interlocutrice principale du gouvernement en ce qui a trait au développement minier au Nunavik) et avec la corporation foncière de la communauté. Enfin, pour les activités d'exploration minière situées en terres de catégorie 3, la consultation doit s'effectuer avec Makivik et, selon le cas, avec toute corporation foncière touchée par les activités. Le promoteur doit par la suite négocier une entente sur les répercussions et avantages avec ces entités et ce, avant d'obtenir les approbations environnementales (Makivik 2014).

L'évaluation préliminaire des projets est effectuée par la Commission de la qualité de l'environnement Kativik (CQEK), qui est formée de cinq représentants du Québec et quatre membres nommés par l'Administration régionale Kativik (ARK). De plus, le Comité consultatif de l'environnement Kativik (CCEK), formé de représentants fédéral, provincial et inuit, veille à l'application et à l'administration des régimes de protection de l'environnement prévus à la Convention. 


\section{Parc national de la Baie-auX-Feuilles ET CLAIMS MINIERS HISTORIQUES}

Depuis les années 1950, des activités d'exploration minière ont lieu dans la région de l'Ungava. Au moment de la signature de la Convention de la Baie-James et de la création du village d'Aupaluk, les négociateurs gouvernementaux auraient refusé de céder aux Inuits le territoire couvert par les titres miniers en le désignant comme des terres de catégorie 3 plutôt que de catégorie 1, pour lesquelles les droits des Inuits auraient été plus importants (Nungak 2017). Aupaluk est donc l'un des seuls villages du Nunavik bordé en partie par des terres de catégorie 3, sur lesquelles le développement minier est permis. Les Aupalumiut ont exprimé leur frustration à l'égard de cette situation dans le rapport Parnasimautik (2014), dans lequel ils proposent de renégocier l'emplacement des terres de catégorie 1 et 2 pour trois villages, dont Aupaluk. Le rapport Parnasimautik est le fruit des consultations tenues dans les villages du Nunavik afin d'élaborer une vision commune du développement régional qui soit conforme à l'identité et à la culture inuit. Il fait suite au rapport «Plan Nunavik », lancé en 2010, en réponse au Plan Nord de Jean Charest. Les Aupalumiut proposent également la création de zones tampons autour des terres de catégorie 1 afin de limiter ou d'interdire le développement minier à proximité des villages.

Une autre demande en ce sens a été faite lors des consultations entourant la création du parc national de la Baie-aux-Feuilles. En effet, la région d'Aupaluk est reconnue pour sa faune abondante - elle abrite plus de quatorze espèces de mammifères marins et une rivière à saumons - et pour son intérêt écologique, notamment parce qu'on peut observer dans la baie Hopes Advance les plus hautes marées du monde (ARK 2015). En 2012, un groupe de travail chargé de délimiter l'aire d'étude du parc et auquel participaient des représentants d'Aupaluk, a demandé à ce qu'une portion de la fosse du Labrador, comprenant les gisements de fer et de nickel convoités par l'industrie, soit ajoutée à l'aire d'étude du parc, car cet endroit représentait un important site de reproduction pour les salmonidés. Les représentants d'Aupaluk ont également signifié leur intérêt pour que le territoire situé aux environs de leur communauté soit inclus dans la superficie du parc. Or, on peut lire dans le rapport que leur « demande n'a pu être retenue étant donné l'importante quantité de titres miniers actifs dans cette région » (ARK $2015: 7$ ).

\section{L'INDUSTRIE ET LES REGISTRES SILENCIEUX DU POUVOIR}

\section{Le projet Hopes Advance}

Le projet Hopes Advance, de la compagnie Oceanic Iron Ore, est un projet de mine de fer qui serait situé à environ $25 \mathrm{~km}$ du village d'Aupaluk et comprendrait trois mines à ciel ouvert. Celles-ci permettraient d'exploiter cinq millions de tonnes de concentré de fer par année durant les cinq premières années d'exploitation, production qui augmenterait par la suite jusqu'à dix millions de tonnes de concentré annuellement (Oceanic Iron Ore 2019). La durée de vie du projet est estimée à vingt-huit ans. Le projet nécessiterait la construction d'un port en eau profonde dans la baie pour acheminer le minerai en Europe ou en Asie, ainsi que d'un pipeline sous-terrain de $21 \mathrm{~km}$, de routes, d'une ligne de transport d'électricité, d'une centrale électrique sur barge et d'un camp de travailleurs pouvant loger jusqu'à quatre cents personnes. Rappelons que la communauté compte moins de deux cents habitants.

\section{SÉDUIRE DES INVESTISSEURS POTENTIELS : LOBBYSME ET RELATIONS DIPLOMATIQUES}

L'exploitation de la mine nécessite des investissements évalués à plus d'un milliard de dollars. Dans une présentation destinée aux investisseurs, la Compagnie décrit le gisement de Hopes Advance comme une « immense ressource exceptionnelle dans un territoire à faible risque » (Oceanic Iron Ore 2016). En effet, selon Oceanic Iron Ore (2016), « [i]l n'existe aucun risque juridique, politique, environnemental ou autre connu susceptible de nuire de manière importante à l'exploitation éventuelle du gisement ». Pour minimiser ces risques et trouver du financement pour son projet, le promoteur doit "séduire » des investisseurs potentiels et créer un climat politique favorable. Ainsi, en 2011, Oceanic Iron Ore nomme à son conseil d'administration Lawrence Cannon, ancien ministre des Transports de l'Infrastructure et des Collectivités (Dansereau 2012). La même année, dans le contexte du développement du Plan Nord, la Société Makivik confirme son appui à la compagnie Oceanic Iron Ore dans sa demande au Gouvernement du Québec concernant la mise en place d'une ligne de transmission électrique desservant la région de l'Ungava de même que d'un port en eau profonde en service à l'année près du village d'Aupaluk. Tel que mentionné dans un communiqué, ces infrastructures « bénéficieraient aux deux parties et sont hautement prioritaires » (Oceanic Iron Ore 2011b). Dans sa lettre d'intention, le président de la Société Makivik de l'époque a déclaré : "Notre relation avec Oceanic a été très fructueuse jusqu'à maintenant et j'aimerais, au nom de Makivik et des Inuits du Nunavik, appuyer entièrement l'initiative Oceanic. » (Oceanic Iron Ore 2011b)

En 2013, le ministère des Finances et de l'Économie du Québec a signé une lettre d'intention à Oceanic Iron Ore pour un investissement dans le projet Hopes Advance, via le fonds d'investissement Capital Mines Hydrocarbures. Par ailleurs, la compagnie est inscrite au registre des Lobbyistes du Québec (2018) avec le mandat suivant :

Démarches en vue de l'attribution d'une subvention ou d'un avantage pécuniaire pour l'implantation d'infrastructures portuaires et le financement d'un quai, un quai à remorqueur et une chaussée 
nécessaires au développement et à l'exploitation du projet minier Hopes Advance situé dans la baie d'Ungava.

En 2016, pour faciliter le financement du projet et aider à la conclusion d'ententes avec les producteurs d'acier chinois, Oceanic Iron Ore signe une entente avec Sinosteel et Power China International, une société d'État chinoise spécialisée dans les travaux de génie civil dans le secteur énergétique. La société chinoise mobilise une équipe de vingt-cinq employés pour faire l'examen du projet Hopes Advance (Oceanic Iron Ore 2017). Dans un communiqué, Oceanic Iron Ore souligne que les démarches conjointes de Power China et de SNC-Lavalin constitue un brillant exemple de coopération bilatérale entre entreprises et nations, telle que souhaitée par le Président Xi et le Premier ministre Trudeau durant le sommet du G20, qui s'est tenu à Hangzhou à l'automne 2016. En effet, dans le cadre de la récente décision du gouvernement canadien de contribuer à la Banque asiatique d'investissement pour les infrastructures, Power China a proposé le projet Hopes Advance au programme du sommet "One Belt One $\operatorname{Road}^{2}$ », qui a eu lieu en mai 2017 à Beijing (Oceanic Iron Ore 2017).

\section{Convaincre la communauté et LES INVESTISSEURS EN DEVENANT 《 UN MODÈLE POUR L'INDUSTRIE »}

Oceanic Iron Ore aspire à développer le projet Hopes Advance selon « un nouveau modèle, basé sur l'acceptabilité sociale ». Le promoteur veut en effet « établir la norme en fonction de laquelle les futurs projets extractifs seront évalués » et soutient que ses «activités futures doivent améliorer la vie de toutes les parties prenantes » (Oceanic Iron Ore 2015). Il propose ainsi de générer du développement économique durable pour améliorer les conditions de vie des communautés locales, notamment en termes de logement et d'éducation, le tout en respectant la culture et les traditions et en minimisant les impacts environnementaux. Enfin, sur son site web, Oceanic s'engage «à faire des relations communautaires une priorité, voire un enjeu organisationnel » (Oceanic Iron Ore 2019 : en ligne).

Les données présentées dans les sections suivantes sont tirées des entretiens qui ont été menés dans la communauté d'Aupaluk en novembre 2017. Bien que ne représentant pas l'opinion de tous les membres du village, elles nous permettent de mieux comprendre certaines de leurs préoccupations et d'évaluer, dans quelle mesure, le projet Hopes Advance s'inscrit dans un modèle « basé sur l'acceptabilité sociale », tel que souhaité par le promoteur.

\section{acceptabilité sociale du projet Hopes Advance}

\section{RISQUES ANTICIPÉS EN RAISON DE LA PROXIMITÉ DU PROJET AVEC LE VILLAGE}

Lors de notre enquête, plusieurs participants s'inquiètent devant l'ampleur des projets proposés et leur proximité avec le village ( $25 \mathrm{~km}$ pour le projet Hopes Advance et $20 \mathrm{~km}$ pour le projet Hawk Ridge). Une participante évoque la particularité des projets miniers aux alentours d'Aupaluk: "Je ne suis pas en faveur du développement minier dans cette communauté, je ne suis pas contre l'exploitation minière en général, mais je pense que la communauté est trop petite et que les projets sont trop proches. Et ce serait trop, trop de terres seraient détruites. » (entrevue avec Mina ${ }^{3}$, 2017) Une autre participante témoigne de ses inquiétudes quant aux impacts des dommages environnementaux sur la pratique d'activités traditionnelles : " J'ai entendu dire qu'ils détruisent la terre, voilà ce que j'ai entendu. Je ne veux pas perdre ma culture. Cueillir des baies, pêcher, faire du camping. J'ai besoin d'aller à la pêche, de cueillir des baies. Ce sont mes activités préférées. » (entrevue avec Kitty, 2017) Une autre participante croit que les dommages environnementaux l'empêcheront de transmettre sa culture à ses enfants :

\footnotetext{
J'ai l'impression qu'ils vont détruire ma terre. Là où j'ai grandi. À mon avis, j'ai un avenir avec mes enfants [...] Je veux vraiment leur apprendre nos coutumes, comment aller chasser, mais ils vont la détruire. Et après l'avoir détruit, comment vais-je parler à mes enfants de nos traditions, de notre culture? Ça me fait très mal parfois. (entrevue avec Rhoda, 2017)
}

Un autre participant mentionne les impacts de la construction d'un port en eau profonde sur les membres de la communauté, surtout en hiver, où la présence de brise-glace les empêcherait de voyager sur la banquise en motoneige et de se rendre dans les villages voisins :

Aupaluk est reconnu pour sa pêche et sa chasse et si... Même les gens qui sont en faveur du projet minier, ils ne veulent pas du port en eau profonde et d'un navire qui va venir tous les jours, tous les jours de l'année, briser la glace. Nous traversons la baie tout le temps en hiver, c'est notre route pour la chasse et la pêche. Donc, s'il y a un navire là-bas, un port en eau profonde là-bas, et que du gaz et du pétrole s'écoulent du navire, toutes les palourdes, les moules et les poissons que nous mangeons seront contaminés. Donc, c'est la chose la plus effrayante. (entrevue avec Henry, 2017)

À noter toutefois que, bien que l'étude de faisabilité du projet publiée en 2012 prévoyait le recours aux briseglaces pour expédier le minerai tout au long de l'année, une nouvelle étude publiée en 2019 mentionne que le transport maritime du minerai serait plutôt réalisé sur une base saisonnière et ce, afin de réduire les coûts et limiter les risques du transport hivernal (Oceanic Iron Ore 2019).

Henry s'inquiète également de la poussière engendrée par la mine et compare la situation à celle de Schefferville, qui possède aussi une mine de fer : "Le vent va souffler de la poussière, de la poussière de minerai de fer... à Schefferville, tout est couvert de poussière rouge et ce sera la même chose ici. » Il mentionne également le bruit, qui sera amplifié par la proximité de la mine : «Les explosions, nous les entendrons, ils ne seront pas si loin, et les camions vont rouler toute la journée et toute la nuit. » Lors des consultations publiques réalisées dans le cadre du rapport Parnasimautik, un participant d'Aupaluk a souligné 
comment les impacts du développement minier se feraient davantage sentir à Aupaluk que dans les deux autres communautés actuellement impactées par la mine Raglan au Nunavik: « Kangiqsujuaq et Salluit ne sont pas touchés par le développement minier comme nous le serons ici à Aupaluk. Les résidents de ces villages ne voient pas et n'entendent pas les activités minières de chez eux contrairement à ce qui va se passer ici. » (Parnasimautik 2014 : 248)

La proximité de la mine avec le village entraîne aussi des inquiétudes liées à la sécurité alimentaire. En effet, en raison du coût de la vie excessivement élevé au Nunavik - notamment causé par les coûts du transport de la nourriture et des matériaux de construction - plus de $37 \%$ des Inuits vivraient sous le seuil de la pauvreté, un taux trois fois plus élevé que celui observé dans le reste du Canada et au Québec (ibid.). La sécurité alimentaire est en donc un enjeu majeur et dépend largement de la capacité des gens à avoir accès à de la nourriture traditionnelle, telle que le caribou, l'omble chevalier, la perdrix, le phoque, le morse et le béluga. Lalimentation traditionnelle permet aussi aux Inuits de rester connectés à leur culture et à leur identité (ibid.). À Aupaluk, certains résidents s'inquiètent que la présence d'une mine à proximité du village ne fasse fuir les animaux :

Parfois, nous n'avons pas de nourriture et nous mangeons du caribou, des poissons, des phoques, tout cela. Une fois que la mine sera en exploitation, est-ce que cela affectera les animaux? Est-ce qu'ils vont continuer de venir? Je n'en suis pas sûr. Je veux que les animaux continuent de venir pour qu'on puisse manger. (entrevue avec Robbie, 2017)

Si la proximité des activités minières avec le village est source d'inquiétude pour les Aupalumiut, notamment en ce qui concerne la sécurité alimentaire, cet élément constitue également un argument en faveur du projet, comme nous le verrons dans la section suivante.

\section{BÉnéfices atTendus des activités d'EXPLOItATION Minière}

Pour certains membres de la communauté, les emplois et les redevances issus des activités minières représentent une planche de salut qui leur permettra de mieux subvenir aux besoins de leur famille: "Les gens mangeraient tous les jours. Les gens manquent de nourriture tout de suite après avoir reçu leur chèque de paie, la nourriture ne dure pas longtemps. Avoir une mine va permettre aux gens de manger tous les jours. » (entrevue avec Elisapi, 2017) Les emplois à la mine constituent également un attrait pour les gens du village: "Il y aura plus d'emplois quand le projet minier va commencer et ça va aider. » (entrevue avec Mary, 2017) Un autre participant mentionne : « Ceux qui s'opposent au projet sont ceux qui travaillent déjà. » (entrevue avec Charlie, 2017) En effet, il semble y avoir une corrélation entre le fait d'être sans emploi et d'être en faveur du développement minier dans la région, corrélation qui apparaît plus clairement dans le sondage effectué par Makivik en février $2015^{4}$. En effet, certains membres de la communauté ayant participé aux travaux de forage des compagnies minières il y a quelques années nous ont affirmé ne pas s'être retrouvé du travail depuis ou n'occuper qu'un emploi à temps partiel (conduire le camion-citerne la fin de semaine, par exemple). Par ailleurs, certains individus en faveur du développement minier ont souligné la précarité économique avec laquelle ils devaient composer et ce, même si aucune question ne portait directement sur cet aspect. Voir à titre d'exemple l'échange suivant :

Considérez-vous qu'Aupaluk est une communauté en santé? Voudriez-vous qu'elle reste telle quelle ou qu'elle change ${ }^{5}$ ?

— Je voudrais qu'elle change, que les gens ne soient plus pauvres.

(entrevue avec Elisapi, 2017)

La position précaire de certains individus en faveur du développement minier pourrait les amener à minimiser les impacts du projet. Ainsi, une participante mentionne : «La migration, les caribous, les poissons... Certaines personnes ne veulent pas du projet. Mais les effets ne se feront pas sentir tout de suite. Ça va prendre des années avant d'affecter la migration [des caribous] et tout...» (entrevue avec Mary, 2017). Elle ajoute ensuite que «les mineurs sont les bienvenus ici ». Les gens au statut économique précaire peuvent aussi avoir peur de critiquer le projet ou de paraître trop exigeants pour ne pas faire fuir la Compagnie. En effet, dans ce type de relation, le rapport de force est clairement en faveur du promoteur, et plutôt que de lui imposer des conditions à respecter, les gens vont plutôt lui demander de l'aide :

Je voudrais savoir, si le projet va de l'avant, est-ce que la Compagnie va aider la communauté ? Par exemple, la nourriture est très chère à la coopérative et nous n'avons qu'un seul moyen de transport, I'aéroport. Vont-ils pouvoir nous aider à obtenir plus de choses dont nous avons besoin? (entrevue avec Rhoda, 2017)

Toutefois, pour cette participante, malgré le manque de ressources de la communauté, un afflux massif d'argent ne semble pas la solution idéale:

Il y a déjà beaucoup de problèmes sociaux ici. Et je ne crois pas que le fait d'avoir une mine va régler ces problèmes. Je suis sûre que les gens auraient plus d'argent grâce aux redevances, mais c'est une communauté très pauvre ici, il n'y a pas beaucoup d'emplois et encore moins des emplois dans la fonction publique avec de bons avantages. Ce sera donc une situation où les gens vont recevoir de l'argent pour ne rien faire - pas pour ne rien faire, mais... On se fera donner la lune, mais on sera payés pour perdre une partie de nous-mêmes. (entrevue avec Mina, 2017)

\section{SCEPTICISME À L'ÉGARd des ACTIVITÉs de Forage ET ÉTUDES ENVIRONNEMENTALES BÂCLÉES}

Les opérations de forage d'Oceanic ont été problématiques et ont donné lieu à plusieurs déversements, comme en témoignent certains membres de la communauté :

Avec la compagnie Forage G4 qui travaillait avec Oceanic, il y a eu plein de déversements partout et ils s'en fichent, l'essence et le 
pétrole... Les gens revenaient en disant que tout cela allait retourner dans la rivière et ils disaient: "Non, non, non, ce n'est pas de I'essence et du pétrole, c'est juste une huile naturelle comme I'huile Crisco mélangée avec les roches rouges. " C'était un mélange rouge très dégoûtant, mais ils ont dit que ce n'était pas mauvais pour I'environnement ${ }^{6}$. (entrevue avec Henry, 2017)

Un membre de la communauté qui a participé aux forages n'a pas apprécié son expérience en raison des déversements, qu'il compare à de la peinture :

J'ai participé aux activités d'exploration, au forage, oui j'en faisais partie, mais je n'aimais pas la façon dont ils le faisaient parce que c'était comme une peinture, comme des cannes de peinture qui étaient renversées sur le sol. [...] Après, je ne suis plus retourné travailler. Je n'ai pas aimé leur façon de travailler. (entrevue avec Charlie, 2017)

À l'été 2014, Oceanic a commencé les études environnementales de référence (baseline studies) avec la firme Genivar, qui a fait l'inventaire de la faune et de la flore qui seraient menacées par le projet. En 2016, le promoteur a réalisé une étude sur les oiseaux migrateurs vulnérables et a complété sa collecte de données axées sur les mammifères marins, les poissons, la qualité de l'eau et l'habitat des mollusques (Oceanic Iron Ore 2019). En entretien, un participant mentionne que ces études environnementales ont été faites à la hâte, sans tenir compte des saisons pour observer la présence des animaux : « Ils ont étudié les oiseaux quand les oiseaux n'étaient plus là. Ils ont étudié les caribous quand ils n'étaient plus là. Ils sont venus à la mauvaise saison pour étudier... Donc c'est un peu effrayant. » (entrevue avec Henry, 2017)

Les doutes quant à la crédibilité des études réalisées par le promoteur ont incité la communauté à entreprendre, en collaboration avec la Société Makivik, une étude d'utilisation du territoire et des connaissances traditionnelles afin de bien identifier les enjeux et les endroits sensibles du territoire qui pourraient être incompatibles avec le développement minier. Cette étude pourra servir de référence advenant que le projet aille de l'avant et qu'il nécessite des études d'impacts sociaux et environnementaux.

\section{Confiance envers le promoteur ET LES REPRÉSENTANTS POLITIQUES}

La section suivante permettra de mieux comprendre les obstacles rencontrés par les membres de la communauté d'Aupaluk lors de leurs échanges avec le promoteur et leurs représentants politiques.

\section{Confiance INTERACTIONNELLE: DES PROCESSUS DE CONSULTATION ET DE COMMUNICATION INADÉQUATS}

S'il y a bien eu une forme de consultation des membres de la communauté par le gouvernement fédéral avant le début des travaux d'exploration, une participante émet des doutes quant à son efficacité :

Il y a quelques années, il y a eu... ils ont appelé ça des consultations, mais elles n'étaient pas très inclusives... Donc, une personne du gouvernement fédéral est venue, a fait des consultations dans la communauté, mais ils n'ont pas amené d'interprète et n'ont pas réussi à en trouver un sur place. Donc ils ont fait cette présentation, et la moitié des gens dans la salle ne parlaient ni anglais ni français... Donc je ne crois pas que l'information est disséminée de façon à ce que tout le monde puisse la comprendre. (entrevue avec Mina, 2017)

Par ailleurs, malgré la création d'un groupe de travail minier qui comprend des représentants de la communauté d'Aupaluk et de la Société Makivik, la communication entre Oceanic Iron Ore et la communauté est difficile. Souvent, les communiqués de presse destinés aux investisseurs constituent les seules sources d'information disponibles. Or, ces documents visent surtout à rassurer les investisseurs et laissent croire que le projet se concrétisera rapidement alors que ce n'est pas le cas, ce qui ne manque pas de semer la panique au village:

En novembre dernier, il y a eu tous ces communiqués de presse sur Oceanic et SNC Lavalin, qui se sont réunis pour créer le plan de la mine, des bâtiments, de la route, et la date limite était novembre. Et tout le monde a vu ça et a paniqué en disant qu'ils étaient prêts, qu'ils se préparaient, qu'ils faisaient les plans et tout. (entrevue avec Henry, 2017)

Non seulement Oceanic Iron Ore concentre ses efforts de communication sur les actionnaires au détriment de la communauté, mais ses représentants ont aussi refusé de rencontrer le maire du village lors d'une visite du site minier avec des investisseurs chinois, ce qui a laissé ce participant perplexe :

\begin{abstract}
II y a deux ans, ils sont arrivés avec un avion rempli de Chinois, un hélicoptère est venu pour les conduire à différents endroits. Et quand je suis allé là-bas, j'ai dit: "Vous allez nous rencontrer? Rencontrer le maire? » II a dit : «Non, non, non, nous n'avons pas le temps de vous rencontrer aujourd'hui. » J'ai dit : "Quoi ? Vous êtes venus jusqu'ici avec les Chinois et vous ne prendrez pas une demi-heure pour aller rencontrer le maire? » " Non, si le maire veut me rencontrer, il peut venir me voir à l'aéroport avant le décollage. » Et des trucs comme ça. De toute façon, ils ont une façon un peu folle de faire les choses, Oceanic.
\end{abstract}

(entrevue avec Henry, 2017)

\section{Confiance INSTItUTIONNELle : pOSITION AMBIGUË DE MAKIVIK PAR RAPPORT AU PROJET}

La Société Makivik a lancé sa politique minière en 2014, dans laquelle elle "réitère son soutien au développement minier durable au Nunavik », dans la mesure où les Inuits du Nunavik «tirent d'importants avantages sociaux et économiques » de ces activités et à condition qu'elles soient réalisées conformément aux lois provinciales et fédérales (Makivik Corporation 2014). Certains membres de la communauté constatent toutefois que la position des dirigeants de la Société est ambiguë en ce qui a trait au projet Hopes Advance. Plusieurs rumeurs circulent, de même que des messages contradictoires, qui varient selon les changements de leadership chez Makivik. 
D'un côté, on dit aux Aupalumiut que le développement minier est inévitable et que leur village sera relocalisé, et de l'autre, qu'on demandera à la Compagnie de déplacer ses opérations plus loin du village :

\begin{abstract}
Le vice-président Développement Économique était Peter Alaku à l'époque pour Makivik et nous entendions différentes rumeurs qui disaient: "Nous ne pouvons pas l'arrêter, ça ira de l'avant et ils pourraient même, vous savez, acheter la communauté et la relocaliser ou offrir un million de dollars à chaque personne et dire : "Allez à Kangirsuk, allez à Tasuijaq, allez ailleurs." Nous allons prendre le contrôle d'Aupaluk et ça va être tellement mauvais pour la communauté que personne ne voudra rester de toute façon [...] Nous allons relocaliser la communauté. La compagnie minière paiera pour que tout le village d'Aupaluk soit déplacé à mi-chemin vers Kangirsuk où les impacts de la mine ne se feront pas sentir. » Ce sont de grosses rumeurs, n'est-ce pas?
\end{abstract}

L'autre extrême, c'est que Makivik va nous appuyer et parler à la Compagnie: "Vous pouvez peut-être déplacer vos activités, même s'il y a beaucoup de minerai de fer à côté d'Aupaluk, il y en a aussi beaucoup jusqu'à Kangirsuk, déplacez vos activités plus loin d'Aupaluk. » [...] Donc, des messages très différents... Cela crée des problèmes, dépendamment à qui vous parlez, les différents représentants régionaux, y compris nos dirigeants locaux, personne ne sait qui croire et c'est très stressant d'avoir une telle réponse d'un côté et une telle autre réponse de l'autre...

(entrevue avec Henry, 2017)

Lambiguité autour de la position de la Société Makivik de même que les nombreuses rumeurs autour du projet entraînent un sentiment d'impuissance vis-à-vis du développement minier, que plusieurs se sentent contraints d'accepter: " Je crois que nous n'avons pas vraiment le pouvoir de dire «non», surtout parce que les organisations régionales sont en faveur des projets et parce que la communauté est si petite que nous avons un très petit impact sur le reste de la région. » (entrevue avec Mina, 2017) Pour cette participante, les enjeux économiques au niveau régional diminuent considérablement le pouvoir d'influence des Aupalumiut : «À l'échelle régionale, la plupart des gens appuient la mine parce qu'elle rapporterait de l'argent à toute la région. Et parce que le gouvernement du Québec s'efforce vraiment de développer le Nord. »

\section{IMPACTS SUR LA COHÉSION SOCIALE ET LA CAPACITÉ D'EXPRESSION}

\section{LOCATION DE MAISONS EN CATIMINI}

La première année durant laquelle la Compagnie a mené ses travaux, elle a déclaré ne pas avoir les moyens ni le temps de bâtir son propre camp, si bien qu'elle a loué près du quart des maisons du village pour loger ses employés. Cette situation a aggravé la pénurie de logements dans le village - pénurie qui touche le Nunavik plus que toute autre région au Québec - forçant les gens à se relocaliser chez des proches, ce qui a contribué au surpeuplement ${ }^{8}$ des logements. Par ailleurs, la Compagnie a aussi fait fi de la procédure l'Office municipal d'habitation Kativik (OMHK) en ce qui concerne la location de maison et payait directement les individus en échange de leur maison. Comme les montants donnés variaient d'une personne à l'autre, la jalousie s'est vite installée entre les gens du village:

\begin{abstract}
Oceanic payait cette famille un tel montant et cette famille un autre, peu importe ce qu'elle acceptait, elle la payait. Et les gens disaient " Ils ont reçu plus d'argent». Et les gens ont découvert qu'ils avaient été payés moins que tout le monde. C'était la pagaille, alors pendant un an, la Compagnie a occupé dix des quarante maisons du village, un quart des maisons, pour mener leurs opérations. (entrevue avec Henry, 2017)
\end{abstract}

La Compagnie était prête à recommencer l'année suivante si ce n'était des demandes répétées de la communauté pour qu'elle bâtisse son propre camp. Or, la communauté n'était pas au bout de ses peines, car une fois son camp bâti sur des terres de catégorie 1, la Compagnie a refusé de s'acquitter de ses obligations financières.

On se dispute toujours avec eux à propos de ça. Ils n'ont payé que trois ans sur les cinq années où ils ont occupé un camp sur des terres de catégorie 1. Et ils ne veulent pas renégocier ${ }^{9}$, alors ils occupent des terres de catégorie 1 depuis des années sans rien payer et ils sont tellement fauchés qu'ils ne peuvent même pas payer le minimum que nous chargerions à une entreprise pour un conteneur. (entrevue avec Henry, 2017)

Au Nunavik, les corporations foncières sont propriétaires des terres de catégorie 1 (droits de surface uniquement) au profit de leurs membres et sont chargées de négocier l'indemnisation à payer pour l'utilisation des droits miniers sur ces terres.

\section{Conflits au sein du leadership}

Les projets miniers à Aupaluk ravivent certaines rivalités familiales, qui se manifestent notamment en conflits entre la mairie et la corporation foncière. Ces tensions prennent parfois de telles proportions qu'elles affectent l'état de santé des individus impliqués:

\footnotetext{
Ils ont tous les deux dit à la radio ici : "Je suis malade, je suis nerveux, nous devons arrêter de nous disputer comme ça, nous devons trouver une façon de nous entendre ». Mais ils n'arrivent pas à s'entendre. Et ils ont pleuré, tous les deux à la radio, en disant essentiellement: "Ma santé se détériore, je n'arrive pas à manger, à dormir, ce projet minier me rend fou, tu me rends fou. » (entrevue avec Henry, 2017)
}

En plus de leur incidence sur la santé des individus, ces conflits nuisent également à la tenue de discussions au sujet des projets au sein de la communauté, comme l'indique une participante:

Il y a quelques années, le support aux projets miniers aurait semblé plus important, parce que les gens ne se sentaient pas à l'aise de s'y opposer ouvertement en raison de la position du leadership de la communauté, qui s'affichait en faveur du développement 
minier. Mais, maintenant, surtout au niveau municipal, le leadership a changé et est davantage opposé aux mines que le précédent. Les gens ont moins peur de subir des préjudices s'ils expriment leur opposition au projet. (entrevue avec Mina, 2017)

À noter à ce propos que la situation se retrouve dans les deux sens. Ainsi, une participante se disant en faveur des projets miniers à Aupaluk nous a mentionné se sentir plus à l'aise de discuter des projets avec la Société Makivik qu'avec le leadership de la communauté.

\section{MÉfIANCE À L'ÉGARD DES MEMBRes DE LA CORPORATION FONCIÈRE}

Les quelques individus dans la communauté qui disposent de plus de détails sur les activités minières, tels que les membres de la corporation foncière, suscitent de la méfiance chez les autres membres de la communauté. En effet, parce qu'ils ont assisté à des séances d'information sur les projets, on les accuse d'être « biaisés » et d'être en faveur de la mine, ce qui nuit encore davantage aux débats et aux discussions au sein du village :

\section{Mon problème, c'est que j'ai parfois l'impression de faire beaucoup de réunions sur le développement minier, mais quand j'en parle à la radio, j'ai parfois l'impression d'aider les compagnies simplement en essayant de l'expliquer. Ils doivent savoir ceci et cela. Je dois dire cela... Parfois, ils sont contre moi, mais nous faisons de notre mieux pour la collectivité. (entrevue avec Louisa, 2017)}

Si la radio demeure le meilleur moyen pour informer les membres de la communauté, il est parfois difficile pour certains d'entre eux de s'exprimer publiquement à propos des projets miniers, comme en témoigne cette participante : «Ils en parlaient souvent à la radio, je voulais dire quelque chose mais j'étais comme muette, je ne pouvais rien dire. Parce qu'on ne peut pas les arrêter et qu'on ne peut rien faire. Je vais perdre mon temps si j'essaie de faire quelque chose. » (entrevue avec Rhoda, 2017) Elle ajoutera plus loin dans l'entrevue:

- Habituellement, quand quelque chose me chicote, quand quelqu'un dit quelque chose qui me dérange, je dois intervenir, dire quelque chose. Mais [pour les mines], je ne dis pas vraiment ce que je pense. Je ne peux pas, je suis un peu gênée [rires].

- Je comprends.

- Je parle beaucoup à la radio, par exemple en ce qui concerne les offres d'emplois, mais je ne sais pas pourquoi je ne parle pas du développement minier lorsqu'ils en parlent...

(entrevue avec Rhoda, 2017)

\section{Tensions ENTRE LES JeUnes Et Les AîNÉs}

La présence de titres miniers historiques à Aupaluk génère un sentiment d'impuissance chez les membres de la communauté, qui croient que le développement minier fait partie de la « destinée » du village, comme en témoigne cette participante : " Je ne pense pas qu'on a le pouvoir de dire «non» parce que [les activités minières] sont là depuis les années 1950. Mon père m'en a déjà parlé, il m’a dit «un jour il y aura une mine ici». Il m'en a parlé quand j'étais encore une petite fille.» (entrevue avec Rhoda, 2017) En plus de contribuer à l'impression « de ne pas avoir le choix », le long historique minier de la région creuse un fossé entre les aînés de la communauté et les jeunes. Ainsi, les aînés sont fiers que leurs parents aient participé aux activités d'exploration dans la région et voient dans les activités minières une occasion pour la communauté de faire des gains financiers considérables. Étant donné la place importante occupée par les aînés dans la culture inuite et l'autorité qu'ils inspirent, cette situation place les plus jeunes dans une position difficile, comme en témoignent ces deux participants :

Nous n'avons pas l'impression de pouvoir consentir ou non, surtout parce qu'il s'agit de titres miniers historiques, établis depuis les années 40 ou 50. Les gens ont été élevés, la génération plus âgée a été élevée en croyant qu'il y aura un projet minier ici un jour, donc... (entrevue avec Mina, 2017)

Ce sont les aînés de la communauté qui disent aux jeunes générations: "Vous devez avoir cette mine, on l'attend depuis longtemps ». Et ce sont les jeunes qui disent: "Nous n'avons pas besoin de cette mine, nous voulons continuer à chasser et à pêcher comme vous l'avez fait, nous voulons que nos enfants... " C'est donc l'inverse de ce que les aînés autochtones disent aux jeunes partout ailleurs dans le monde : "Sauver la terre ». Alors, c'est très étrange. Donc, à cause de cela, il y a aussi des tensions. Les jeunes qui disent : "On ne veut pas de ça ». Tu es censé respecter tes aînés... Et les anciens disent : " Mais vous devez l'avoir ». Donc c'est fou ici, c'est beaucoup de tensions. (entrevue avec Henry, 2017)

Certains aînés, conscients de la position des jeunes, se sentent toutefois impuissants :

— On en parle depuis 2009-2010 [...] Ils disaient : « Ne détruisez pas ma rivière, c'est là où je vais pêcher. »Certaines personnes ont pleuré parce qu'ils ne veulent pas d'eux ici. Les jeunes sont passés par toute une gamme d'émotions. Ils ne veulent pas de la mine ici, mais on n'avait pas le choix quand ils ont commencé... On en parle depuis tellement d'années.

- Et pensez-vous que vous auriez le pouvoir de stopper le projet si vous le vouliez?

- On participe à plusieurs rencontres, surtout concernant la création du parc national. Mais on ne peut rien faire parce que c'est déjà décidé. Ils ont déjà les claims, on ne peut pas faire grand-chose

(entrevue avec Louisa, 2017)

Cette position ne reflète toutefois pas l'opinion de tous les aînés du village, comme en témoigne cette participante, à qui nous avons posé la question suivante:

- Pensez-vous que les Inuits ont le pouvoir de choisir quel genre de projets doit être réalisé dans leur région?

- Quand la communauté est unie et en accord sur certains sujets, comme les mines, par exemple, oui, ils ont le pouvoir. Ils ont le pouvoir. (Martha, 2017) 


\section{Conclusion}

Malgré la fierté associée à l'héritage minier de la région, la légitimité du projet Hopes Advance semble mitigée. En effet, dans les années 1970, le processus de négociation qui a mené à la désignation de terres de catégories 3 aux abords du village d'Aupaluk a généré beaucoup de frustration. Un litige qui pourrait être apaisé par la création de zones tampons autour des terres de catégorie 1 afin de limiter ou d'interdire le développement minier à proximité des villages (Parnasimautik 2014). Certains représentants de la communauté ont également manifesté le désir que leur village soit inclus dans la superficie du parc national de la Baie-aux-Feuilles (ARK 2015).

Si les Aupalumiut ont utilisé les ressources institutionnelles à leur disposition pour faire entendre leurs préoccupations, le promoteur lui, dispose d'un important registre de pouvoir «silencieux» (Frederiksen et Himley 2019). En effet, celui-ci tente de séduire des investisseurs potentiels et de créer un climat politique favorable au moyen de campagnes de lobbysme et de relations publiques au Québec et en Chine, en désignant le projet Hopes Advance comme une "immense ressource exceptionnelle dans un territoire à faible risque » (Oceanic Iron Ore 2016). Par ailleurs, bien que le promoteur veuille s'ériger en « modèle pour l'industrie », les négociations secrètes entourant la location de maison, le non-respect de ses obligations financières liées à son camp d'exploration, les études environnementales bâclées, le manque d'information au sujet du projet et le peu d'égard témoigné aux représentants du village lors d'une visite de la région avec des investisseurs chinois ont quelque peu entaché sa crédibilité. Bien que certains Aupalumiut n'hésitent pas dénoncer publiquement ses agissements ${ }^{10}$, d'autres, aux prises avec un statut précaire, semblent prêts à lui donner une chance dans l'espoir d'améliorer leurs conditions de vie.

Enfin, les nombreuses rumeurs sur une possible relocalisation du village, avec ou sans l'appui de Makivik, rumeurs qui ont circulé selon les changements de leadership au sein de la Société, ont contribué à ébranler la confiance de certains membres de la communauté à l'égard de leurs représentants, en plus de générer un sentiment d'impuissance. La capacité d'expression et de délibération des Aupalumiut est aussi limitée par les conflits au sein du leadership et la méfiance à l'égard des membres de la corporation foncière. Enfin, la présence de claims miniers historiques suscite des tensions entre les jeunes et les aînés, lesquels constituent une figure d'autorité dans la culture inuite.

Le cas d'Aupaluk, bien que connu dans la région du Nunavik, a bénéficié d'une couverture médiatique faible dans le reste du Québec, comparativement à d'autres projets controversés. Si on ne peut conclure qu'un projet est accepté socialement sur la base d'une absence de conflits ou de protestations ouvertes à son sujet, du moins à l'échelle provinciale, le cas d'Aupaluk nous enseigne que pour mieux saisir les dimensions « silencieuses » de l'acceptabilité sociale, il faut simplement tendre une oreille plus attentive.

\section{Notes}

1. Cet article présente les résultats d'une recherche réalisée dans le cadre d'une thèse de doctorat en communication publique intitulée "Linfluence des communautés cries et inuites dans l'évaluation et l'autorisation des projets miniers dans le nord du Québec : quelle place pour le consentement libre, préalable et éclairé? » Cette recherche a été menée en collaboration avec la Corporation foncière d'Aupaluk et la Société Makivik. Les résultats ont été présentés à la communauté d'Aupaluk en avril 2018 via une émission de radio interactive. Ils ont également été présentés au Nunavik Mining Workshop 2018, qui s'est tenu à Kuujjuaq du 24 au 26 avril 2018.

2. Instauré par le Président chinois Xi, le projet «One Belt One Road », aussi désigné comme " Nouvelle route de la soie », sera constitué de routes terrestres et maritimes qui relieront la Chine aux anciennes républiques de l'Union soviétique, au Pakistan, à l'Inde et au reste de l'Asie du Sud-Est. Le projet, le plus gros de l'histoire, selon certains analystes, nécessitera des investissements de près d'un trillion de dollars à travers le monde (Chatzky et McBride 2019; Phillips 2017).

3. Nom fictif.

4. Le sondage réalisé par la Société Makivik en février 2015 auprès de 80 personnes de la communauté (41 femmes et 39 hommes) a montré que $65 \%$ des Aupalumiut se disaient contre le développement minier dans leur communauté, alors que 29 \% des répondants croyaient qu'une mine pouvait générer des bénéfices pour la communauté, surtout en ce qui a trait aux emplois. Â noter que 29 \% des répondants s'étaient aussi déclarés sans emploi.

5. À noter que les participants qui s'opposaient aux projets miniers ont plutôt souligné qu'Aupaluk était une communauté en santé et qu'ils ne voulaient pas qu'elle grandisse trop vite.

6. Une situation qui rappelle le cas de Tata Steel Minerals Canada, dont les installations sont situées à Schefferville, et qui a fait l'objet d'une plainte émise par le Conseil innu de Matimekush-Lac John, appuyé par un biologiste indépendant, à propos de vingt-trois déversements d'eaux rouges dans les cours d'eau avoisinant ses opérations. La minière soutient qu'en dépit de leur couleur, les déversements ne sont pas nocifs pour l'environnement. Le dossier fait présentement l'objet d'une enquête par le Directeur des poursuites criminelles et pénales (Lachapelle-Plamondon et Habel-Thurton 2019).

7. Nom fictif.

8. La pénurie de logements au Nunavik a des conséquences sur la santé physique et mentale des Nunavimmiut, particulièrement les enfants, et contribue à l'accroissement de la violence domestique et sexuelle (Makivik 2010).

9. Au moment d'écrire ces lignes, le village d'Aupaluk a la responsabilité du démantèlement du camp d'exploration d'Oceanic Iron Ore, un litige qui perdure entre le village et la Compagnie, qui refuse d'assumer les coûts de réexpédition du matériel laissé sur place.

10. Voir à ce propos l'article « Là où la terre est rouge », dans lequel Janice Grey, une jeune élue locale, explique que 
« [1]e consentement préalable, libre et éclairé, n’a jamais vraiment été un droit dont Aupaluk a pu bénéficier» (Labrecque-Saganash 2018).

\section{Ouvrages cités}

ALLEN, John, 2003 : Lost geographies of power. Blackwell, Oxford, UK.

ARK (Administration régionale Kativik), 2015 : Projet de Parc national de la Baie-aux-Feuilles : état des connaissances. Administration régionale Kativik, Service des ressources renouvelables, de l'environnement, du territoire et des parcs, Section des parcs, Kuujjuaq, Québec.

BATELLIER, Pierre, 2015 : Acceptabilité sociale : cartographie d'une notion et de ses usages. Cahier de recherche | 2015 UQAM : Les publications du Centr'ERE.

BOURGEOIS, Sabrina, 2017 : Comprendre la construction du « moratoire administratif» sur l'exploration/l'exploitation uranifère: L'influence des coalitions allochtones et autochtones. Mémoire de maîtrise, département de science politique, Université Laval, Québec.

CAMPBELL, Bonnie, et Marie-Claude PRÉMONT, 2017 : «What is behind the search for social acceptability of mining projects? Political economy and legal perspectives on Canadian mineral extraction ». Miner Economics 30 : 171-180.

CARON-MALENFANT, Julie, Michel VENNE et Maxime BEAUCAGE, 2013 : Étude sommaire sur les processus et les facteurs d'acceptabilité sociale pour le secteur industriel. Institut du Nouveau Monde, Montréal. <https://archives.bape.gouv.qc.ca/sections/mandats/mine_apatite_sept-iles/documents/DC11.pdf> (consulté le 26 sept. 2019).

CCEK (Comité consultatif de l'environnement Kativik), 2016 «Évaluation environnementale applicable au projet de mine de fer Hopes Advance (Nunavik, Québec)». Lettre adressée à l'Administratrice provinciale - Convention de la Baie-James et du Nord québécois, 25 février.

CHATZKY, Andrew, et James MCBRIDE, 2019 : China's Massive Belt and Road Initiative. 21 may 2019, Council on Foreign Relations. <https://www.cfr.org/backgrounder/chinas-massive-belt-androad-initiative> (consulté le 14 décembre 2019).

CPEQ (Conseil patronal de l'environnement du Québec), 2012 : Guide des bonnes pratiques afin de favoriser l'acceptabilité sociale des projets. Montréal.

DANSEREAU, Suzanne, 2012 : «Québec doit-il investir dans Oceanic Iron Ore? » Lesaffaires.com, Dossier: Grand Nord. 11 février. <https://www.lesaffaires.com/dossier/grand-nord/quebec-doit-il-investir-dans-oceanic-iron-ore/540756> (consulté le $1^{\mathrm{er}}$ octobre 2018).

DAYMON, Christine, et Immy HOLLOWAY, 2010 : Qualitative Research Methods in Public Relations and Marketing Communications. Second Edition, Routledge, New York.

DESHAIES, Michel, 2011 : « Grands projets d'exploitation minière et stratégie des firmes pour se rendre environnementalement acceptables ». L'Espace politique 15(2011-3). <https://journals.openedition.org/espacepolitique/2113> (consulté le 16 janvier 2020).

FORTIN, Marie-José, et Yann FOURNIS, 2015 : «Une définition territoriale de l'acceptabilité sociale: pièges et défis conceptuels ». VertigO - la revue électronique en sciences de l'environnement 15(3). <https://journals.openedition.org/vertigo/16682> (consulté le 16 janvier 2020).
FREDERIKSEN, Tomas, et Matthew HIMLEY, 2019: "Tactics of dispossession: Access, power, and subjectivity at the extractive frontier». Transactions of the Institute of British Geographers: 1-15. <https://doi.org/10.1111/tran.12329> (consulter le 16 janvier 2020)

GAUTHIER, Mario, 2015: Évaluations environnementales stratégiques sur les hydrocarbures: Analyse des facteurs d'influence de l'acceptabilité sociale des activités de mise en valeur des hydrocarbures et propositions relatives au mode de gouvernance territoriale. Centre de recherche sur la gouvernance des ressources naturelles et des territoires (CRGRNT), novembre.

GENDRON, Corinne, 2014 : «Penser l'acceptabilité sociale : au-delà de l'intérêt, les valeurs ». Communiquer : Revue de communication sociale et publique 11 : 117-129.

GUIKAWUIHÉ GNABOA, Romuald, 2018 : L'acceptabilité sociale comme une nouvelle norme à atteindre pour l'adhésion de plusieurs types d'acteurs dans un projet minier: Cas du projet d'exploitation d'uranium par la firme Strateco à Matoush dans les territoires des Cris. Mémoire de maîtrise, Université d'Ottawa, Ottawa.

KOIVUROVA, Timo, et al., 2015 : " "Social license to operate": A relevant term in Northern European mining? »Polar Geography 38(3) : 194-227.

LABRECQUE-SAGANASH, Maïté, 2018 : «Là où la terre est rouge ». Métro, 6 décembre. <https://journalmetro.com/ opinions/maitee-labrecque-saganash/1967183/la-ou-la-terreest-rouge-1-2/> (consulté le 14 décembre 2019).

LACHAPELLE-PLAMONDON, Nicolas, et Djavan HABELTHURTON, 2019 : " Les eaux rouges de Tata Steel font des vagues jusqu'à l'Assemblée nationale ». Radio-Canada, 29 mai. <https://ici.radio-canada.ca/nouvelle/1172631/eaux-rouges-tatasteel-mine-quebec> (consulté le 14 décembre 2019)

LANDRY, Angie, et Karine MATEU, 2017: "La requête de Ressources Strateco rejetée par la Cour supérieure du Québec ». Radio-Canada, 21 juin. <https://ici.radio-canada.ca/nouvelle/1041095/la-requete-de-ressources-strateco-rejetee-parla-cour-superieure-du-quebec> (consulté le 9 décembre 2019).

LE BLANC, Kelly, 2009: Évaluation de la participation des Cris dans la procédure d'évaluation environnementale de la Convention de la Baie James et du Nord québécois (CBJNQ). Mémoire de maîtrise, département de géographie, Université de Montréal, Montréal.

MAILLÉ, Marie-Ève, 2012 : Information, trust, and social cohesion in an environmental conflict related to a wind farm project in Quebec (Canada). Thèse de doctorat, département de communication sociale et publique, Université du Québec à Montréal, Montréal.

MAKIVIK CORPORATION, 2010 : «Serious housing shortage in Nunavik - Inuit sounds a warning to federal government». Communiqué de presse, 17 mars.

—, 2014 : « Nunavik Inuit Mining Policy ». <https://www.makivik. org/wp-content/uploads/2014/11/NMP-ief-F.pdf> (consulté le 11 décembre 2019).

MRN (Ministère de l'Énergie et des Ressources naturelles), 2016 : Orientations du ministère de l'Énergie et des Ressources naturelles en matière d'acceptabilité sociale (livre vert). Ministère de l'énergie et des ressources naturelles, Québec.

NUNGAK, Zebedee, 2017 : Wrestling with Colonialism On Steroids: Quebec Inuit Fight for Their Homeland. Véhicule Press, Montréal.

OCEANIC IRON ORE CORP., $2011 \mathrm{la}$ : « Oceanic Iron Ore Corp. Agrees Letter of Intent with Inuit of Nunavik ». Communiqué de presse, 4 août. 
_, 2011b : «Makivik Corporation Supports Oceanic in Respect of Infrastructure Development ». Communiqué de presse, 20 septembre.

—, 2014 : «Oceanic Provides Corporate Update». Communiqué de presse, 18 août.

—, 2015: «Oceanic President \& CEO Announces Intention to Develp Hopes Advance Employing a New Model Based on Social Acceptability ». Communiqué de presse, 10 novembre.

—, 2016 : Présentation aux investisseurs. Août. 48 p.

—, 2017 : «Oceanic Announces Receipt of Power China EPC Proposal and Provides Corporate Update ». Communiqué de presse, 31 mars.

—, 2019 : « Social and Community Considerations ». <http://oceanicironore.com/company/social-community-considerations/> (consulté le 14 décembre 2019).

—, 2019 : «Oceanic Announces Results of a PEA Study». Communiqué de presse, 19 décembre.

PARNASIMAUTIK CONSULTATION REPORT, 2014: On the Consultations Carried out with Nunavik Inuit in 2013. Rapport, 14 novembre. <https://www.cerp.gouv.qc.ca/fileadmin/Fichiers_ clients/Documents_deposes_a_la_Commission/P-202.pdf> (consulté le 16 janvier 2020)

PHILLIPS, Tom, 2017 : «The $\$ 900$ bn question: what is the Belt and Road initiative? » The Guardian, 12 mai. <https://www.theguardian.com/world/2017/may/12/the-900bn-question-what-isthe-belt-and-road-initiative> (consulté le 14 décembre 2019).

PIEKKARI, Rebecca, C. WELCH et E. PAAVILAINEN-MÄNTYMÄKI, 2009: "The Case Study as Disciplinary Convention». Organizational Research Methods 12(3) : 567-589.

PRNO, Jason, et Scott SLOCOMBE, 2012 : «Exploring the origins of "social license to operate" in the mining sector: Perspectives from governance and sustainability theories ». Resources Policy 37(3) : 346-357.

RADIO-CANADA, 2019: « Le droit au froid : le cri d'alarme du peuple inuit ». Radio-Canada | Médium large 14 février. Sur Internet (consulté le 11 décembre 2019).

REGISTRE DES LOBBYISTES DU QUÉBEC, 2018 : Sommaire de l'inscription no E18-LE00773 de l'entreprise Oceanic Iron Ore Corp. Ministère de la Justice, Registre des Lobbyistes du Québec. (consulté le $1^{\text {er }}$ octobre 2018).

SAUVÉ, Lucie, et Pierre BATELLIER 2011 : « La mobilisation citoyenne sur la question du gaz de schiste au Québec : une exigence de démocratie ». Nouveaux Cahiers du Socialisme $6:$ 224-236.

STEVENSON, Marc G., 1993: Traditional Inuit Decision-Making Structures and the Administration of Nunavut. The Royal Commission on Aboriginal Peoples, Ottawa.

THÉRIAULT, Sophie, 2010 : « Repenser les fondements du régime minier québécois au regard de l'obligation de la Couronne de consulter et d'accommoder les peuples autochtones». McGill International Journal of Sustainable Development Law and Policy 6(2): 217-245.

THOMSON, Ian, et Robert G. BOUTILIER, 2011 : « Social license to operate », in P. Darling (dir.), SME Mining Engineering Handbook: 1779-1796. Society for Mining, Metallurgy and Exploration, Littleton, $\mathrm{CO}$

WOLSINK, Maarten, 2000 : « Wind power and the NIMBY-myth: Institutional capacity and the limited significance of public support ». Renewable Energy 21(1) : 49-64.

ZAHARA, Alexander, Arn KEELING et Trevor BELL, 2016 : Social Licence to Operate: Background and State of Knowledge Report. Report prepared for the Memorial Dialogue on Social Licence to Operate, Memorial University of Newfoundland, 12 septembre. 